International Journal of Pure and Applied Mathematics

Volume 102 No. 4 2015, 733-745

ISSN: 1311-8080 (printed version); ISSN: 1314-3395 (on-line version)

url: http://www.ijpam.eu

doi: http://dx.doi.org/10.12732/ijpam.v102i4.11

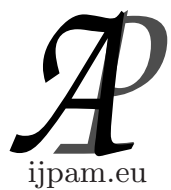

\title{
SIMPLE MOTION PURSUIT DIFFERENTIAL GAME OF MANY PURSUERS AND ONE EVADER ON CONVEX COMPACT SET
}

\author{
Idham Arif Alias ${ }^{1}$, Raja Noorsuria Raja Ramli ${ }^{2}$, \\ Gafurjan Ibragimov ${ }^{3}$, Anvar Narzullaev ${ }^{4}$ \\ ${ }^{1,2,3}$ Institute for Mathematical Research and Department of Mathematics \\ Faculty of Science \\ Universiti Putra Malaysia \\ 43400 UPM Serdang, Selangor, MALAYSIA \\ ${ }^{4}$ Faculty of Computer Science and Information Technology \\ Universiti Putra Malaysia \\ 43400 UPM Serdang, Selangor, MALAYSIA
}

\begin{abstract}
We study a differential game of many pursuers and single evader in nonempty closed bounded convex subset of $\mathbb{R}^{n}$. In this game, all players cannot leave the given set. Control parameters of all players are subjected to geometric constraints. Maximum speeds of all players are equal to 1. Pursuit is said to be completed if geometric position of at least one pursuer coincides with that of the evader. Pursuers try to complete the pursuit. Problem is to find estimate for guaranteed pursuit time. To solve the problem, first, we study the same problem in an $n$-dimensional cube. Then, we reduce the main problem to the game in the cube. To this end, we use method of fictitious pursuers. In this paper, we improve the estimate for guaranteed pursuit time from $O\left(n^{3}\right)$ to $O\left(n^{2}\right)$.
\end{abstract}

AMS Subject Classification: 49N70, 93C95

Key Words: differential game, control, strategy, state constraint

Received: $\quad$ March 29, 2015

(c) 2015 Academic Publications, Ltd. url: www.acadpubl.eu 


\section{Introduction}

Differential game is a field that has particular interest in constructing the most favorable strategies for all players. A large number of papers were devoted to differential game of many pursuers and one evader or several evaders (see, for example [1] -[21]). Simple motion differential game of many pursuers attract the attention of many researchers (see, for example, [1] -[19] and [21]). Among them, papers [3], [4], [8], [10], [15] and [18] are devoted to games with geometric constraints.

Pshenichnii[18] was one of the early researchers who studied simple motion differential game of many pursuers and one evader. In this game, maximum speeds of all players are equal to 1 . It was proved that if the initial position of the evader $y_{0}$ belongs to the interior of convex hull of the initial positions of $m$ pursuers, $x_{10}, \ldots, x_{m 0}$, that is, $y_{0} \in \operatorname{int} \operatorname{conv}\left\{x_{10}, \ldots, x_{m 0}\right\}$, then pursuit can be completed, otherwise evasion is possible.

Chikrii and Prokopovich[8] found sufficient solvability conditions of pursuit and evasion for the simple motion differential game of a group of pursuers and one evader. The game is described by the following differential equations,

$$
\dot{z}_{i}=u_{i}-v, \quad z_{i} \in \mathbb{R}^{k}, \quad u_{i} \in U_{i}, \quad v \in V, \quad z_{i}(0)=z_{i}^{0}, \quad i=1, \ldots, n,
$$

where $\mathbb{R}^{k}, U_{i}$ and $V$ are nonempty compact sets. A family of nonempty convex compacta $M_{1}, \ldots, M_{n}$ are given in $\mathbb{R}^{k}$, defining the terminal set $M^{*}$.

Chernous'ko[4] solved a simple motion evasion differential game of many pursuers and one evader. Here maximum speed of the evader is assumed to be equal to $\alpha>1$, whereas maximum speeds of all pursuers are equal to 1 . It was shown that evasion is possible from any finite number of pursuers. Result of this work further was extended to a wide class of problems by Chernous'ko and Melikyan[5], and Chernous'ko and Zak [6].

Blagodatskikh and Petrov [3] obtained necessary and sufficient conditions of completion of pursuit in a simple motion differential game of many pursuers and many evaders, provided that all the evaders use the same control, and the maximum speeds of all players are equal to 1 . It should be noted that the papers [10] and [15] were studied under the state constraint.

Kuchkarov et. al[15] solved a problem of simple motion pursuit-evasion differential game that involved $m$ pursuers and single evader on the surface of 
a given cylinder. The movements of all players are described by equations,

$$
\begin{aligned}
P_{i} & : \quad \dot{x}_{i}=u_{i}, \quad x_{i}(0)=x_{i 0}, \quad\left|u_{i}(t)\right| \leq 1, i=1,2, \ldots, m, \\
E & : \quad \dot{y}=v, \quad y(0)=y_{0}, \quad|v(t)| \leq 1,
\end{aligned}
$$

where $x_{i}, x_{i 0}, y, y_{0}, u_{i}, v \in \mathbb{R}^{3}$. Two cases were considered in this paper: (a) all pursuers move freely and unbounded with any phase constraint (b) the movement of all pursuers bounded on the surface of the cylinder. Meanwhile, the evader always moves on the surface of a given cylinder $M$, for both cases, where $M$ is described by the following equation,

$$
M=\left\{q=\left(q_{(1)}, q_{(2)}, q_{(3)}\right) \mid q_{(1)}^{2}+q_{(2)}^{2}=R^{2}, q_{(3)} \in \mathbb{R}\right\} .
$$

Furthermore, the pursuers apply a counter strategy to guarantee pursuit while the evader applies a positional strategy to ensure evasion. In this paper, it was proved that if there exist some $i, j \in\{1,2, \ldots, m\}$ such that $x_{i 0(3)}<y_{0(3)}<$ $x_{j 0(3)}$, then pursuit can be completed for some time $T$ in both cases (a) and (b). However, if $i, j \in\{1,2, \ldots, m\}$ do not satisfy the condition $x_{i 0(3)}<y_{0(3)}<x_{j 0(3)}$, then evasion is possible for both cases.

Ivanov[10] studied a pursuit-evasion differential game of many pursuers and one evader in a compact set. All players have the same dynamical possibilities and motions of all players are described by equations,

$$
\dot{x}_{i}=u_{i}, x_{i}(0)=x_{i 0},\left|u_{i}\right| \leq 1, i=0,1,2, \ldots, m,
$$

where $x_{i}, u_{i} \in \mathbb{R}^{n}, u_{i}, i=1,2, \ldots m$, are control parameters of the pursuers and $u_{0}$ is control parameter of the evader. During the game, all players must not leave a given compact convex subset $A$ of $\mathbb{R}^{n}, n \geq 2$. It is assumed that $A$ has nonempty interior. It was proved that if the number pursuers less than the dimension of the space $(m<n)$, then evasion is possible. If $m \geq n$, then it was proved that pursuit can be completed. Moreover, in this paper guaranteed pursuit time $T$ is estimated by a third degree polynomial in $n$, more precisely $T \leq\left(n^{3}-2 n^{2}+n+1\right) d$, where $d$ is the diameter of $A$.

In the present paper, we also study a differential game of many pursuers and single evader in nonempty closed bounded convex subset $A$ of $\mathbb{R}^{n}, n \geq 2$. Similar to the game studied by Ivanov[10], all players cannot leave the given set $A$ and control parameters of all players are subjected to geometric constraints. We will improve the estimation of guaranteed pursuit time given by Ivanov[10]. 


\section{Statement of the problem}

We consider a pursuit differential game described by the following equations:

$$
\begin{aligned}
P_{i} & : \quad \dot{x}_{i}=u_{i}, \quad x_{i}(0)=x_{i 0}, \quad\left|u_{i}\right| \leq 1, \quad i=1,2, \ldots, m, \\
E & : \quad \dot{y}=v, \quad y(0)=y_{0}, \quad|v| \leq 1,
\end{aligned}
$$

where $x_{i}, y, u_{i}, v, x_{i 0}, y_{0} \in \mathbb{R}^{n}, m \geq n$.

Definition 1. A measurable function $u_{i}(\cdot)=u_{i}(t), t \geq 0$, is called a control of the pursuer $x_{i}$ if $\left|u_{i}(t)\right| \leq 1, t \geq 0$, and the solution $x_{i}(\cdot)=x_{i}(t)$, $t \geq 0$, of the initial value problem $\dot{x}_{i}=u_{i}(t), x_{i}(0)=x_{i 0}, i \in\{1,2, \ldots, m\}$, satisfies the inclusion

$$
x_{i}(t) \in A .
$$

Definition 2. A measurable function $v(\cdot)=v(t), t \geq 0$, is called a control of the evader $y$ if $|v(t)| \leq 1, t \geq 0$, and the solution $y(\cdot)=y(t), t \geq 0$, of the initial value problem $\dot{y}=v(t), y(0)=y_{0}$, satisfies the inclusion

$$
y(t) \in A .
$$

Let $H(0,1)$ be the ball of radius 1 and centered at the origin.

Definition 3. A function $U_{i}\left(x_{i}, y, v\right), U_{i}: \mathbb{R}^{n} \times \mathbb{R}^{n} \times H(0,1) \rightarrow H(0,1)$, is called strategy of the pursuer $x_{i}$ if for any control of the evader $v(t)$ the initial value problem

$$
\begin{aligned}
\dot{x}_{i} & =U_{i}\left(x_{i}, y, v(t)\right), & x_{i}(0)=x_{i 0}, \\
\dot{y} & =v(t), & y(0)=y_{0},
\end{aligned}
$$

has a unique absolutely continuous solution $\left(x_{i}(t), y(t)\right), t \geq 0$, with $x_{i}(t), y(t) \in$ $A, t \geq 0$.

Definition 4. If $x_{i}(\tau)=y(\tau)$ at some $i \in\{1,2, \ldots, m\}$ and $\tau \geq 0$, then we say that pursuit is completed at the time $\tau$ (by the pursuer $x_{i}$ ).

Definition 5. A number $T$ is called guaranteed pursuit time if there exist strategies of pursuers $U_{1}, U_{2}, \ldots, U_{m}$ such that for any control of the evader $v(\cdot)$ an equality $x_{i}(\tau)=y(\tau)$ holds at some $i \in\{1,2, \ldots, m\}$ and $0 \leq \tau \leq T$ where $\left(x_{i}(t), y(t)\right)$, is the solution of (3), (4) at $v=v(t), t \geq 0$.

During the pursuit, all players may not leave the given set $A$. The aim of pursuers is to minimize the guaranteed pursuit time, and that of the evader is 
to maximize it.

Problem. Find a guaranteed pursuit time estimated from above by a second degree polynomial in $n$ and construct strategies of the pursuers.

\section{The main results}

In this section, we obtain a guaranteed pursuit time. First, we study the problem in the case where the set $A$ is a cube.

\subsection{Differential game on cube}

We first consider an auxiliary differential game when $m=n$ and

$$
A=N=\left\{\left(q_{1}, q_{2}, \ldots, q_{n}\right) \mid 0 \leq q_{i} \leq a, i=1,2, \ldots, n\right\},
$$

where $a$ is a positive number. Clearly, $N$ is an $n$-dimensional cube with edges equal to $a$. Thus, all the players move in $N$.

Lemma 6. In the differential game on $N$, the number

$$
T=\frac{a}{2}\left(n^{2}-n+\sqrt{n}+1\right)
$$

is a guaranteed pursuit time.

Proof. We construct the strategies of pursuers as follows. Let

$$
u_{i}(t)=\frac{2\left(\xi_{0}-x_{i 0}\right)}{a \sqrt{n}}, \quad 0 \leq t \leq t_{1}, \quad i=1,2, \ldots, n, \quad t_{1}=\frac{a \sqrt{n}}{2},
$$

where $\xi_{0}=\left(\frac{a}{2}, \frac{a}{2}, \ldots, \frac{a}{2}\right)$ is the center of the cube $N$. According to (5), each pursuer moves towards the point $\xi_{0}$ on the time interval $\left[0, t_{1}\right]$. Clearly,

$$
x_{i}\left(t_{1}\right)=\xi_{0},
$$

that is, all the pursuers reach the point $\xi_{0}$ at the time $t_{1}$. 
Next, we define strategies of the pursuers on the time interval $\left(t_{1}, t_{1}+\frac{a}{2}\right]$ as follows:

$$
\begin{aligned}
& u_{1}(t)=\left(0, u_{1,2}(t), 0, \ldots, 0\right), \\
& u_{2}(t)=\left(0,0, u_{2,3}(t), \ldots, 0\right), \\
& \ldots \ldots \ldots \ldots \ldots \ldots \ldots \ldots \ldots \ldots \ldots \ldots \ldots \ldots \ldots, \\
& u_{n-1}(t)=\left(0, \ldots, 0,0, u_{n-1, n}(t)\right), \\
& u_{n}(t)=\left(u_{n, 1}(t), 0,0, \ldots, 0\right),
\end{aligned}
$$

where

$$
\begin{aligned}
& u_{i, i+1}(t)=\left\{\begin{array}{rr}
1, \quad y_{i+1}\left(t_{1}\right)>\frac{a}{2}, \\
-1, \quad y_{i+1}\left(t_{1}\right)<\frac{a}{2},
\end{array} \text { if } t<\tau_{i+1}^{1},\right. \\
& u_{i, i+1}(t)=v_{i+1}(t), \quad t \geq \tau_{i+1}^{1}, \quad i=1, \ldots, n,
\end{aligned}
$$

where $\tau_{i+1}^{1}$ is a time for which $x_{i, i+1}\left(\tau_{i+1}^{1}\right)=y\left(\tau_{i+1}^{1}\right)$. In the sequel, we use notations $y_{n+i}=y_{i}, x_{i, n+j}=x_{i, j}, v_{n+i}=v_{i}$.

Let us illustrate the part of strategy defined in (6)-(8) for $i=1$, that is, for the pursuer $x_{1}$. If $y_{2}\left(t_{1}\right)>\frac{a}{2}(\operatorname{see}(7))$, then $u_{1,2}(t)=1$, and so the pursuer $x_{1}$ moves with the speed equal to 1 parallel to $q_{2}$-axis in positive direction of $q_{2}$-axis. Since the evader may not leave the set $N$, therefore $y_{2}\left(\tau_{2}^{1}\right)=x_{1,2}\left(\tau_{2}^{1}\right)$ at some $\tau_{2}^{1} \in\left(t_{1}, t_{1}+\frac{a}{2}\right]$. Then according to $(8), u_{1,2}(t)=v_{2}(t), t \geq \tau_{2}^{1}$, which guarantees $x_{1,2}(t)=y_{2}(t)$, for all $t \geq \tau_{2}^{1}$.

In the case $y_{2}\left(t_{1}\right)<\frac{a}{2}$ also using a similar reasoning, we can obtain $x_{1,2}\left(\tau_{2}^{1}\right)=$ $y_{2}\left(\tau_{2}^{1}\right)$ at some $\tau_{2}^{1} \in\left(t_{1}, t_{1}+\frac{a}{2}\right]$, and then the control $u_{1,2}(t)=v_{2}(t)$ guarantees that $x_{1,2}(t)=y_{2}(t)$ for all $t \geq \tau_{2}^{1}$. Thus, using the strategies (6)-(8), we obtain

$$
x_{i, i+1}(t)=y_{i+1}(t), \quad t \geq \tau_{i+1}^{1},
$$


where $\tau_{i+1}^{1} \in\left(t_{1}, t_{1}+\frac{a}{2}\right]$ is some time.

Further, the strategy $u_{i}(t)=\left(u_{i 1}(t), \ldots, u_{i n}(t)\right)$ of the pursuer $x_{i}, i=$ $1,2, \ldots, n$, is constructed as follows. Starting from the time $\tau_{i+1}^{1}$ we let

$$
\begin{aligned}
u_{i, i+1}(t) & =v_{i+1}(t), u_{i, i+2}(t) & = & + \\
u_{i, i+j}(t) & =0, & j & =3,4, \ldots, n,
\end{aligned}
$$

until $x_{i, i+2}(t)=y_{i+2}(t)$. In (9), we take " + " if $x_{i, i+2}\left(\tau_{i+1}^{1}\right)<y_{i+2}\left(\tau_{i+1}^{1}\right)$, and we take " - " if $x_{i, i+2}\left(\tau_{i+1}^{1}\right)>y_{i+2}\left(\tau_{i+1}^{1}\right)$. Part of the strategy defined by (9) ensures that $x_{i, i+1}(t)=y_{i+1}(t)$ since $u_{i, i+1}(t)=v_{i+1}(t)$.

Afterwards, we construct the strategy of $x_{i}$ inductively as follows. Let,

$$
x_{i, i+j}(t)=y_{i+j}(t), \quad j=1,2, \ldots,(k-1),
$$

and pursuer $x_{i}$ be moving according to the strategy

$$
u_{i}(t)=\left(u_{i, 1}(t), u_{i, 2}(t), \ldots, u_{i, n}(t)\right), \quad t \geq \tau_{i+k-1}^{k-1},
$$

until $x_{i, i+k}(t)=y_{i+k}(t)$ with

$$
\begin{aligned}
& u_{i, i+1}(t)=v_{i+1}(t), \quad u_{i, i+2}=v_{i+2}(t), \quad \ldots, \quad u_{i, i+k-1}(t)=v_{i+k-1}(t), \\
& u_{i, i+k}(t)={ }_{-}^{+} \sqrt{1-v_{i+1}^{2}(t)-v_{i+2}^{2}(t)-\ldots-v_{i+k-1}^{2}(t)}, \\
& u_{i, i+j}(t)=0, \quad j=(k+1), \ldots, n,
\end{aligned}
$$

where $t=\tau_{i+k-1}^{k-1}$, is the first time for which $x_{i, i+k}(t)=y_{i+k}(t)$.

In (10), “+" is taken if $x_{i, i+k}\left(\tau_{i+k-1}^{k-1}\right)<y_{i+k}\left(\tau_{i+k-1}^{k-1}\right)$, and " - " is taken if $x_{i, i+k}\left(\tau_{i+k-1}^{k-1}\right)>y_{i+k}\left(\tau_{i+k-1}^{k-1}\right)$. If $x_{i, i+k}\left(\tau_{i+k}^{k}\right)=y_{i+k}\left(\tau_{i+k}^{k}\right)$ for the first time at some $\tau_{i+k}^{k}$, then we set

$$
\begin{aligned}
& u_{i, i+1}(t)=v_{i+1}(t), \quad u_{i, i+2}(t)=v_{i+2}(t), \quad \ldots, \quad u_{i, i+k}(t)=v_{i+k}(t), \\
& u_{i, i+k+1}(t)={ }_{-}^{+} \sqrt{1-v_{i+1}^{2}(t)-v_{i+2}^{2}(t)-\ldots-v_{i+k}^{2}(t)}, \\
& u_{i, i+j}(t)=0, \quad j=(k+2), \ldots, n, \quad \tau_{i+k}^{k} \leq t<\tau_{i+k+1}^{k+1},
\end{aligned}
$$


where $t=\tau_{i+k+1}^{k+1}$ is the time for which $x_{i, i+k+1}(t)=y_{i+k+1}(t)$. Note that to obtain equality $x_{i, i+k+1}(t)=y_{i+k+1}(t)$, the pursuer $x_{i}$ on the interval $\left[\tau_{i+k}^{k}, \tau_{i+k+1}^{k+1}\right]$ along $q_{i+k+1}$-axis has to travel the distance equal at most to $\frac{a}{2}$.

Therefore, starting from the time $t_{1}+\frac{a}{2}=\frac{\sqrt{n}+1}{2} a$, each pursuer has to travel the distance equal at most to $\frac{a(n-1)}{2}$ exactly to complete the pursuit. All pursuers altogether have to travel the distance equal to $n \frac{a(n-1)}{2}$. This distance decreases with the rate equal to

$$
\begin{aligned}
\alpha(t) & =\sum_{i \in I_{1}} \sqrt{1-v_{i+1}^{2}(t)}+\sum_{i \in I_{2}} \sqrt{1-v_{i+1}^{2}(t)-v_{i+2}^{2}(t)}+\ldots \\
& +\sum_{i \in I_{\mathrm{n}}} \sqrt{1-v_{i+1}^{2}(t)-v_{i+2}^{2}(t)-\ldots-v_{i+n-1}^{2}(t)}
\end{aligned}
$$

where $I_{1}, I_{2}, \ldots, I_{n}$ are disjunctive subsets of the set $I=\{1,2, \ldots, n\}$, and $I_{1} \cup$ $I_{2} \cup \ldots \cup I_{n}=I$. We estimate $\alpha(t)$ as follows:

$$
\begin{aligned}
\alpha(t) & \geq \sum_{i \in I_{1}} \sqrt{1-v_{i+1}^{2}(t)-v_{i+2}^{2}(t)-\ldots-v_{i+n-1}^{2}(t)}+\ldots \\
& +\sum_{i \in I_{\mathrm{n}}} \sqrt{1-v_{i+1}^{2}(t)-v_{i+2}^{2}(t)-\ldots-v_{i+n-1}^{2}(t)} \\
& =\sum_{i=1}^{n} \sqrt{1-v_{i+1}^{2}(t)-v_{i+2}^{2}(t)-\ldots-v_{i+n-1}^{2}(t)} .
\end{aligned}
$$

Clearly, if $0 \leq x \leq 1$, then $\sqrt{x} \geq x$, and therefore

$$
\begin{aligned}
\alpha(t) & \geq\left(1-v_{2}^{2}(t)-v_{3}^{2}(t)-\ldots-v_{n}^{2}(t)\right)+\left(1-v_{3}^{2}(t)-v_{4}^{2}(t)-\ldots-v_{1}^{2}(t)\right) \\
& +\ldots+\left(1-v_{1}^{2}(t)-v_{2}^{2}(t)-\ldots-v_{n-1}^{2}(t)\right) \\
& =n-(n-1)\left(v_{1}^{2}(t)+v_{2}^{2}(t)+\ldots+v_{n}^{2}(t)\right. \\
& \geq n-(n-1) \\
& =1 .
\end{aligned}
$$


Thus, $\alpha(t) \geq 1$, meaning that the distance $\frac{n(n-1) a}{2}$ is decreasing with the rate $\alpha(t) \geq 1$ and, hence, it will have been traveled by all the pursuers for the time $\frac{n(n-1)}{2} a$. As the consequence, the guaranteed pursuit time in the differential game on $N$ is

$$
T \leq \frac{\sqrt{n}+1}{2} a+\frac{n(n-1)}{2} a=\frac{a}{2}\left(n^{2}-n+\sqrt{n}+1\right) .
$$

The proof of Lemma 6 is completed.

\subsection{General case}

In this section, we consider the differential game on $A$. It is not difficult to construct a cube containing the set $A$. Without any loss of generality, we assume that the cube $N$ defined by

$$
N=\left\{\left(q_{1}, q_{2}, \ldots, q_{n}\right) \mid 0 \leq q_{i} \leq a, i=1,2, \ldots, n\right\}
$$

contains the set $A$. Let $T_{1}=\frac{a}{2}\left(n^{2}-n+\sqrt{n}+1\right)$.

Theorem 7. Pursuit can be completed in the differential game (1)-(2) for the time $T_{1}$.

Proof. Fictitious pursuers. Introduce fictitious pursuers. The fictitious pursuers can move either inside or outside the given closed bounded convex set $A$. However, they move only inside the cube $N$. Motions of the fictitious pursuers are described by the following equations:

$$
\bar{P}_{i}: \dot{\bar{x}}_{i}=w_{i}, \quad \bar{x}_{i}(0)=x_{i 0}, \quad\left|w_{i}\right| \leq 1, \quad i=1,2, \ldots, n,
$$

where $\bar{x}_{i}, w_{i}, \in \mathbb{R}^{n}$.

According to this equation, the initial position of the fictitious pursuer $\bar{x}_{i}$ is the same as that of the pursuer $x_{i}$. The strategies of the fictitious pursuers on $\left[0, T_{1}\right]$ are defined as the strategies of real pursuers in the cube $N$ (as described in the proof of Lemma above) and these strategies ensure the equality $\bar{x}_{i}(\tau)=y(\tau)$ at some $\tau \in\left[0, T_{1}\right]$ and $i \in\{1,2, \ldots, n\}$. Real pursuers can move only inside the given set $A$. Thus, we introduced $n$ fictitious pursuers, whose initial positions coincide with the initial positions of the real pursuers $x_{10}, x_{20}, \ldots, x_{n 0}$, and move in $N$. By Lemma, they can complete the pursuit on 
$\left[0, T_{1}\right]$

Strategies of the real pursuers. Following Ivanov[10], construct a strategy for the pursuer $x_{i}$ by means of the strategy of the fictitious pursuer $\bar{x}_{i}$. Define the control $u_{i}(t)$ of the pursuer $x_{i}$ from the requirement of the equation $x_{i}(t)=$ $F\left(\bar{x}_{i}(t)\right)$, where $F\left(\bar{x}_{i}(t)\right)$ is the projection of the point $\bar{x}_{i}(t)$ on the given set $A$, which is defined as follows:

$$
|x-F(x)|=\min _{a \in A}|x-a| .
$$

It is not difficult to show that the projection function $F(x), x \in \mathbb{R}^{n}$, for the closed convex set $A$ has the following properties:

1. $F(x)=x$, if $x \in A$.

2. $|F(x)-F(y)| \leq|x-y|$ for all $x, y \in \mathbb{R}^{n}$.

3. If $\bar{x}(t), t \geq 0$, is absolutely continuous, then $x(t)=F(\bar{x}(t)), t \geq 0$, is also absolutely continuous.

Since $\bar{x}_{i}(t)$ is absolutely continuous, then according to property 3 of projection so is $x_{i}(t)$. Admissibility of the pursuer's strategy can be shown as in the paper of Ivanov[10].

Proof that pursuit can be completed. Since $\bar{x}_{i}(\tau)=y(\tau)$ at some time $\tau \in\left[0, T_{1}\right]$ and $i \in\{1,2, \ldots, n\}$ and $y(\tau) \in A$, then $\bar{x}_{i}(\tau) \in A$. This implies that $\bar{x}_{i}(\tau)=F\left(\bar{x}_{i}(\tau)\right)=x_{i}(\tau)$ (see Property 1 ). Thus, $y(\tau)=x_{i}(\tau)$. Hence, pursuit is completed as the position of a fictitious pursuer $\bar{x}_{i}$ coincides with the position of the evader $y$.

The proof of Theorem 7 is complete.

\section{Conclusion}

We have studied a differential game of $m$ pursuers and one evader on closed convex set $A$. The case $m<n$, where $n$ is dimension of the space $\mathbb{R}^{n}$, was studied by Ivanov[10] and in this case evasion is possible. We have proved the theorem for $n$ pursuers. Clearly, this implies validity of the theorem for $m \geq n$. 
We have proved that guaranteed pursuit time is estimated by a second degree polynomial in $n$. Now, we can compare values of polynomials for guaranteed pursuit times. From the Figure 1 below, the first column of the table corresponds to Ivanov's guaranteed pursuit time, and the second column corresponds to ours one, where $a$ is edge of the cube that contains the set $A$. We let $a=1$ and obtained the result as follow.

\begin{tabular}{|c|c|c|}
\hline$n$ & $T=a\left(n^{3}-2 n^{2}+n+1\right)$ & $T=\frac{a}{2}\left(n^{2}-n+\sqrt{n}+1\right)$ \\
\hline 1 & 1.00 & 1.00 \\
\hline 2 & 3.00 & 2.21 \\
\hline 3 & 13.00 & 4.37 \\
\hline 4 & 37.00 & 7.50 \\
\hline 5 & 81.00 & 11.62 \\
\hline 6 & 151.00 & 16.73 \\
\hline
\end{tabular}

Figure 1: The values of polynomials for guaranteed pursuit times.

\section{References}

[1] T. G. Abramyantz, E. P. Maslov, A differential game of pursuit of a group target, Izv. Akad. Nauk. Tecr. Sist. Upr., 5, (2004), $16-22$.

[2] P. Borowko, W. Rzymovski, Avoidance of many pursuers in the simple motion case, J. Math. Analys. Appl., 111, (1985), 535 - 546.

[3] A. I. Blagodatskikh, N. N. Petrov, Conflict Interaction Controlled Objects Groups, Udmurt State University, Izhevsk, Russia, (2009), 266. (In Russian).

[4] F. L. Chernous'ko, A problem of evasion from many pursuers, Prikladnaya Matematika I Mekhanika, 40(1), (1976), $14-24$.

[5] F. L. Chernous'ko, A. A. Melikyan, Game-Theoretic Problems of Control and Search, Nauka, Moscow (1978). (In Russian).

[6] F. L. Chernous'ko, V. L. Zak, On differential games of evasion from many pursuers, Journal of Optimization Theory and Applications, 46, No. 4 (1985), 461-470. 
[7] W. Chodun, Differential games of evasion with many pursuers, J. Math. Anal. and Appl., 142, No. 2 (1989), 370 - 389.

[8] A. A. Chikrii, P. V. Prokopovich, Linear avoidance in the case of interaction of controlled objects groups, Annals of the International Society of Dynamic Games, New Trends in Dynamic Games and Applications, Birkhäuser, Boston, 3, (1995), 259 - 269.

[9] N. L. Grigorenko, The problem of pursuit by several objects, Differential Games - Development in Modeling and Computation, Espoo, 156, (1990), Lecture Notes in Control and Inform. Sci., Springer, Berlin, (1991), 71 80.

[10] R. P. Ivanov, Simple pursuit-evasion on the compact, Dokl. USSR., 254, No. 6 (1980), 1318-1321.

[11] R. P. Ivanov, Yu. S. Ledyaev, Optimality of pursuit time in a simple motion differential game of many objects, Trudy Mat. Inst. im. Steklova Akad. Nauk. SSSR., 158, (1981), $87-97$.

[12] G. I. Ibragimov, Optimal pursuit with countable many pursuers and one evader, Differential Equations, Springer, 41(5), (2005), 627 - 635.

[13] G. I. Ibragimov, and N. A. Satimov, Multiplayer pursuit differential game on a closed convex set with integral constraints, Abstract and Applied Analysis, Hindawi Publishing Corporation, 2012, (2012), 1 - 12.

[14] G. I. Ibragimov, M. Salimi, M. Amini, Evasion from many pursuers in simple motion differential game with integral constraints, European Journal of Operational Research, 218, (2012), 505 - 511.

[15] A. Sh. Kuchkarov, M. Risman, A. H. Malik, Differential game with many pursuers when evader moves on the surface of a cylinder, ANZIAM J., 53(E), (2012), E1 - E20.

[16] A. Sh. Kuchkarov, G. I. Ibragimov, M. Khakestari, On a linear differential game of optimal approach of many pursuers with one evader, Journal of Dynamical and Control Systems, 19, No. 1 (2013), 1 - 15.

[17] L. A. Petrosjan, Pursuit games with many participants, Izv. Akad. Nauk. Arm. SSR., 1, No. 5 (1966), $331-340$.

[18] B. N. Pshenichnii, Simple pursuit by several objects, Cybernetics, No. 3 (1976), $145-146$. 
[19] N. N. Petrov, Simple pursuit of rigidly connected evaders, Avtomatika $i$ Telemekhanika, 12, (1997), $89-95$.

[20] D. M. Stipanovic, A. A. Melikyan, N. Hovakimyan, Some sufficient conditions for multi-player pursuit-evasion games with continuous and discrete observations, Annals of the International Society of Dynamics Games, Springer, Berlin, 11: Advances in Dynamic Games and Applications, (2009), $133-145$.

[21] D. A. Vagin, and N. N. Petrov, Simple pursuit of rigidly connected evaders, Izv. Ross. Akad. Nauk. TSU., No. 5 (2001), $75-79$. 
